\title{
Cosmic Far-Infrared Background Fluctuations revealed by the FIRBACK Observing Program
}

\author{
Guilaine Lagache and Jean-Loup Puget \\ Institut d'Astrophysique Spatiale, 91405 Orsay, France
}

\begin{abstract}
We have used the Far-InfraRed BACKground (FIRBACK) field observations with ISOPHOT at $170 \mu \mathrm{m}$ to search for the Cosmic Far-InfraRed Background (CFIRB) fluctuations. Our study is based on power spectra analyses which allow us to statistically separate each of the background components (Galactic and extragalactic). We show that the extragalactic background fluctuations in FIRBACK fields lie well above the instrumental noise and the cirrus confusion noise. The observed power spectra show a flattening at high spatial frequencies which is due to unresolved extragalactic sources. The level of the extragalactic power spectrum fluctuations is nearly the same in all FIRBACK fields. This is the first time that fluctuations due to unresolved extragalactic sources have been isolated at far-infrared wavelengths. In the two largest FIRBACK fields, the power spectra clearly reveal the presence of correlated extragalactic fluctuations, which we are currently analyzing. However, if $3 \sigma$ detected sources are cleaned from the maps, the CFIRB fluctuations appear at this time to be compatible with a Poissonian distribution. We would need an independent cirrus template (like for the HI gas) to more incisively remove the Galactic contribution to see whether additional correlations are present.
\end{abstract}

